\title{
Scan Statistics on Poisson Random Fields with Applications in Genomics
}

\author{
Supplementary Materials
}

\author{
Nancy R. Zhang \\ Benjamin Yakir \\ Li C. Xia \\ David O. Siegmund
}

July 20, 2015

Note: References of the form (\#.\#) in this document refer to equations in the main paper.

\section{Comments on More Complex Types of Struc- tural Variants}

There are other structural variations that we do not model formally. Inversions are a segment of the genome that is read in the reverse direction. There will be hanging reads, essentially indistinguishable from those of a simple insert, so additional evidence is required to make a distinction. There should be a decrease in the depth of coverage; and if the inverted region is enough longer than the read length that some small number of reads originate from completely within the inverted segment of the target genome, the inversion should be easily detected, provided that we ask whether otherwise unmapped reads would map in the reverse direction. A translocation, where a segment of the genome changes its location, represents a deletion in the place vacated and an insertion in its new location. If the insertion is substantially longer 
than the read length, one can expect some of the hanging reads from the insertion to cluster at the location of the deletion.

Our principal model for insertions involves a single inserted segment. Another possibility is a tandem repeat, where a genomic segment is repeated, perhaps because of slippage in recombination. Suppose, for simplicity, there is a single repeat. The insert length statistic in this case is essentially the same as above, but it must span both the original and the repeated segment, which would have a total length of $2 w$, and hence it may be less powerful than it would be for a simple insertion. The hanging read statistic would now be very similar to that for detecting deletions. In the case of deletions the hanging read must originate from a read in the target genome that covers the point of the deletion, whereas for a tandem repeat the hanging read would originate from a read covering the point where the repeats are joined. Also as in the case of deletions, there would be two peaks separated by a distance equal to the width of the insertion. The difference that would distinguish a deletion from an insertions is that in the former case $Z^{-}$must be shifted to the right to align it with the peak from $Z^{+}$, whereas for a tandem repeat, it would be moved to the left.

\section{Moments}

Here, we derive the moments of the likelihood ratio statistic under a measure transformation that are used for the p-value approximations and power calculations. Consider the expectation and variance of (3.4). Let $\mathbb{P}$ be the measure where $N(d z)$ has null intensity $\lambda_{0}(d z)$, and define

$$
d \mathbb{P}_{\tau}=\exp \left(\ell_{\tau}\right) d \mathbb{P}=\exp \left[\beta \int k_{\tau}(z) N(d z)-\psi_{\tau}(\beta)\right] d P .
$$

We can show that under $\mathbb{P}_{\tau}, N(d z)$ is still a Poisson random field but with intensity function (3.3). Let $\mathbb{E}_{\tau}$ and $\operatorname{Var}_{\tau}$ be expectation and variance, respectively, under $\mathbb{P}_{\tau}$. Then, the first two moments of $\ell_{\tau}$ under the formal alternative are

$$
\mathbb{E}_{\tau}\left[\ell_{\tau}\right]=\int\left[\beta k_{\tau}(z) e^{\beta k_{\tau}(z)}-e^{\beta k_{\tau}(z)}+1\right] \lambda_{0}(d z),
$$

and

$$
\operatorname{Var}_{\tau}\left[\ell_{\tau}\right]=\int\left[\beta k_{\tau}(z)\right]^{2} e^{\beta k_{t}(z)} \lambda_{0}(d z)
$$


For the mixture model in Section 3.1, when $k$ is written in the form (3.6), these simplify to

$\mathbb{E}_{\tau}\left[\ell_{\tau}\right]=\int_{t_{1}}^{t_{2}} \rho(t) d t \int[\beta g(y, w, r) \exp [\beta g(y, w, r)]-\exp [\beta g(y, w, r)]+1] d F_{0}(y)$

and a similar expression for the variance.

\subsection{The expectation and covariance structure of the local field}

We call $\ell_{\sigma}-\ell_{\tau}$, for $\sigma$ close to $\tau$, the local field of $\tau$. For p-value approximations we will also need the moments of the local field under $\mathbb{P}_{\tau}$. The expectation is

$$
\mathbb{E}_{\tau}\left(\ell_{\sigma}-\ell_{\tau}\right)=\int\left[\beta\left(k_{\sigma}(u)-k_{\tau}(u)\right) e^{\beta k_{\tau}(u)}-e^{\beta\left(k_{\sigma}(u)\right)}+e^{\beta\left(k_{\tau}(u)\right.}\right] \lambda_{0}(d u) .
$$

For the mixture model where $k$ is in the form (3.6), where $\tau=\left(t_{1}, t_{2}, r, w\right)$ and $\sigma=\left(t_{1}, t_{2}, q, \eta\right)$, let $D_{\sigma}$ denote differentiation with respect to $\sigma$, and let $\dot{k}$ denote partial derivatives of $k$ with respect to $(q, \eta)$. Then we also obtain expressions for the gradient, $D_{\sigma} \mathbb{E}_{\tau}\left(\ell_{\sigma}-\ell_{\tau}\right)$, which vanishes when $\sigma=\tau$. The Hessian evaluated at $\sigma=\tau$ is

$$
\left.D_{\sigma}^{2} \mathbb{E}_{\tau}\left(\ell_{\sigma}-\ell_{\tau}\right)\right|_{\sigma=\tau}=-\beta^{2} \int\left[\dot{k}_{\tau}(u)\right]\left[\dot{k}_{\tau}(u)\right]^{\prime} e^{\beta k_{\tau}(u)} \lambda_{0}(d u)=-\Sigma_{\tau},
$$

say, which also equals the negative of the covariance function of $\dot{\ell}_{\tau}=\beta \int \dot{k}_{\tau}(u) N(d u)$.

Suppose the values of $w, r$ and $\beta$ are held fixed and the values of $t_{1}$ and $t_{2}$ are allowed to vary. Consider the two parameter sets

$$
\tau=\left(t_{1}, t_{2}, w, r, \beta\right), \quad \sigma=\left(t_{1}+\epsilon_{1}, t_{2}+\epsilon_{2}, w, r, \beta\right) .
$$

The expectation of the difference is

$$
\mathbb{E}_{\tau}\left(\ell_{\sigma}-\ell_{\tau}\right)=\frac{\epsilon_{2}^{+}+\epsilon_{1}^{-}}{t_{2}-t_{1}} \mathbb{E}\left[\ell_{\tau}\right]-\frac{\epsilon_{2}^{-}+\epsilon_{1}^{+}}{t_{2}-t_{1}} \mathbb{E}_{\tau}\left[\ell_{\tau}\right]
$$

and the variance is

$$
\operatorname{Var}_{\tau}\left(\ell_{\sigma}-\ell_{\tau}\right)=\frac{\epsilon_{2}^{+}+\epsilon_{1}^{-}}{t_{2}-t_{1}} \operatorname{Var}\left[\ell_{\tau}\right]+\frac{\epsilon_{2}^{-}+\epsilon_{1}^{+}}{t_{2}-t_{1}} \operatorname{Var}_{\tau}\left[\ell_{\tau}\right]
$$


In the variables $t_{1}, t_{2}$, the local process has independent increments and behaves stochastically like Brownian motion, i.e., a non-differentiable process (see Yakir and Pollak, 1998 and Siegmund, Yakir and Zhang, 2010; intuitively the independence is due to the Markov nature of Poisson processes). In the other parameters the model varies smoothly.

In the special case where $\epsilon_{1}=\epsilon_{2}=\epsilon$ the expectation and variance have the form

$$
\mathbb{E}_{\tau}\left(\ell_{\sigma}-\ell_{\tau}\right)=-\xi_{\tau} \cdot|\epsilon|
$$

and

$$
\operatorname{Var}_{\tau}\left(\ell_{s}-\ell_{\tau}\right)=\varsigma_{\tau}^{2} \cdot|\epsilon|
$$

\section{Approximating the Probability of Crossing a Threshold}

Here, we sketch the approximation method that led to the analytic formulas of Sections 4 and 5 of the paper. Let $T$ be the domain of variation of the scan parameter $\tau$. Consider, for some threshold function $x_{t}, \mathbb{P}\left(\cup_{t \in T}\left\{\ell_{t} \geq x_{t}\right\}\right)$, the probability that the likelihood ratio statistic (or some other suitable scanning statistic) crosses the threshold at some point in $T$. We propose to use the following steps in order to produce an analytical approximation for this probability:

1. Identify the parameter value(s) that maximize the marginal probability:

$$
\tau=\arg \max _{t \in T} \mathbb{P}\left(\ell_{t} \geq x_{t}\right) .
$$

2. Restrict $T$ to the collection of parameter values for which the marginal probabilities are in the same order of magnitude as the maximal marginal probability. For likelihood ratio statistics, this may be all or most of the values of $t$.

3. Apply the measure-transformation technique described in Siegmund, Yakir and Zhang (2011).

The measure transformation technique relies on rewriting the probability of interest:

$$
\mathbb{P}\left(\cup_{t \in T}\left\{\ell_{t} \geq x_{t}\right\}\right)=\sum_{t \in T} e^{-x_{t}} \mathbb{E}_{t}\left[\frac{M_{t}}{S_{t}} e^{-\left[\ell_{t}-x_{t}+m_{t}\right]} ; \ell_{t}-x_{t}+m_{t} \geq 0\right]
$$


with $M_{t}$ and $S_{t}$ being the maximization and summation, respectively, with respect to $s$, of $\exp \left\{\ell_{s}-\ell_{t}+x_{t}-x_{s}\right\}$; and $m_{t}=\log M_{t}$. Note that $M_{t}$ and $S_{t}$ rely only on the local field of $t$, whereas the rest of the quantity within $\mathbb{E}_{t}[\ldots]$ above rely on the "global" field $\ell_{t}$. The localization theorem (Theorem 3.1 of Siegmund, Yakir and Zhang (2011)) states that, under certain conditions, the local and global components are asymptotically independent, which gives

$\lim _{\rho \rightarrow \infty} \rho^{\frac{1}{2}} \mathbb{E}_{t}\left[\left(M_{t} / S_{t}\right) e^{-\left(\ell_{t}-x_{t}+m_{t}\right)} ; \ell_{t}-x_{t}+m_{t} \geq 0\right]=\sigma_{t}^{-1} \phi\left(\mu_{t} / \sigma_{t}\right) \mathbb{E}_{t}\left[\mathcal{M}_{t} / \mathcal{S}_{t}\right]$

where

$$
\mu_{t}=\lim _{\rho \rightarrow \infty}\left[\mathbb{E}_{t}\left(\ell_{t}\right)-x_{t}\right] / \rho^{\frac{1}{2}}, \quad \sigma_{t}^{2}=\lim _{\rho \rightarrow \infty} \operatorname{Var}_{t}\left(\ell_{t}\right) / \rho .
$$

To evaluate $\mathbb{E}[\mathcal{M} / \mathcal{S}]$, we differentiate between the smooth component and the Brownian motion-type component in $\ell_{t}$. For the former the appro-

priate rate parameter is $\rho^{-\frac{1}{2}}$ and for the latter the it is $\rho^{-1}$. To determine the mean of the local field, we take second order Taylor expansion of $\mu_{t}$ with respect to the smooth parameter(s) while holding the Brownian motion-type parameters fixed and then add that to the drift (2) of the Brownian motion type component. It follows that

$$
\mathbb{E}_{t}\left[\mathcal{M}_{t} / \mathcal{S}_{t}\right]=\rho^{-\frac{5}{2}} \times \xi_{t} \nu\left(2 \xi_{t} / \varsigma_{t}^{2}\right) \times(2 \pi)^{-\frac{3}{2}}\left|\Sigma_{t}\right|^{\frac{1}{2}}
$$

where now $\Sigma_{t}=\mathbb{E}_{t}\left(\dot{\ell}_{t} \dot{\ell}_{t}^{\prime}+\ddot{x}_{t}\right)$. The function $\nu$ is defined and a simple approximation given on p. 112 of Siegmund and Yakir (2007). A variation on this method also applies when the kernel $g$ is continuous and vanishes at $\pm \infty$, as in the model (2.2) or the models for hanging reads. The details are omitted here, but some examples are given below.

\section{Special Cases of the Approximations in Sec- tion 4.1}

There are two special cases of particular interest. If the threshold $x_{0}$ does not depend on $w$ and $r$, the equation defining $\beta_{w}$ is $\rho \delta J\left(\beta_{w}, w, r\right)=x_{0}$, so (4.6) specializes to

$$
1-\exp \left\{-m e^{-x_{0}} \rho \int_{w_{0}}^{w_{1}}\left[\xi\left(\beta_{w}\right)-\xi(0)\right] \nu\{\cdot\}[\Sigma(w)]^{1 / 2}\left[\left(2 \pi(\rho \delta)^{1 / 2} \sigma\left(\beta_{w}\right)\right]^{-1} d w\right\} .\right.
$$


In this case $\Sigma(w)$ is most easily computed as the variance of $D_{w} \ell(t ; w, r)$.

The second case of particular interest involves events of the form

$$
\left\{\max _{t, w_{0} \leq w \leq w_{1}} Z(t ; w, r) \geq x_{1}\right\}
$$

which can be written in the form of (4.5) by putting $x_{w, r}=\beta x_{1}-\rho \delta \psi(\beta ; w, r)$. In this case $\beta$ is chosen to satisfy $x_{1}=\mathbb{E}_{\tau}[Z(t, w, r)]$. Although we have calculated the relevant integrals numerically, the Kullback-Leibler information $J$ in (4.6) typically has a minimum value, so the integral can be approximated by Laplace's method. This can be useful in multi- dimensional problems, where numerical integration could be computationally onerous.

\section{Details of Monte Carlo Accuracy Evalua- tions of Section 4.2}

The number of repetitions of the Monte Carlo experiment is 2000 (except for the last two rows, where the number of repetitions was increased to 2500). The threshold $x$ and sample size $m$ are smaller than one might want to use in practice, since a Monte Carlo experiment for more realistic values would be extremely time consuming. The table contains two analytic approximations. For the first we take $\nu=1$, while the second uses the computed value of $\nu$. In almost all cases the second approximation is more accurate, although it is usually still somewhat conservative.

\section{P-value Thresholds for Tables 2, 3, and 4}

In Table 2, for the case of $R=36, \delta=200, \sigma=10, p=0.03$ the 0.05 threshold for $Z^{H}$ is $x_{1}=13.2$. The corresponding threshold for the the statistic based on $Z^{B}$ is $x_{1}=10.4$. For $R=100, \delta=220, \sigma=63, p=0.033$, the threshold for the statistic based on $Z^{H}$ is $x_{1}=15.8$. For $Z^{B}$ it is $x_{1}=0.21$.

In Table 3, for $R=100, \sigma=63$, the 0.05 threshold for $Z^{B}$ about $x_{1}=2.6$; for $Z^{H}$ it is about $x_{1}=10.0$.

The 0.05 thresholds for $Z^{B}$ and $Z^{H}$ in Table 4 of main manuscript are respectively 22.1 and 53.0 . 
Table 1: Approximate p-values

\begin{tabular}{c|c|c|c|c|c|c|c|c|c}
\hline$m$ & $x$ & $\rho$ & $\delta$ & $w$ & $r$ & $\sigma$ & Approx1 & Approx2 & Monte Carlo \\
\hline 1000 & 6.10 & 1 & 40 & 3.0 & 0.1 & 4 & 0.047 & 0.033 & 0.037 \\
1000 & 6.10 & 4 & 40 & 3.0 & 0.1 & 4 & 0.060 & 0.039 & 0.050 \\
1000 & 6.10 & 10 & 40 & 3.0 & 0.1 & 4 & 0.065 & 0.042 & 0.053 \\
1000 & 6.15 & 1 & 20 & 3.0 & 0.1 & 1 & 0.050 & 0.037 & 0.016 \\
2000 & 5.00 & 1 & 200 & 1.5 & 0.1 & 1 & 0.059 & 0.050 & 0.045 \\
2000 & 5.00 & 0.5 & 200 & 1.5 & 0.1 & 1 & 0.052 & 0.045 & 0.042 \\
2000 & 5.00 & 0.25 & 200 & 1.5 & 0.1 & 1 & 0.045 & 0.040 & 0.027 \\
2000 & 5.00 & 0.5 & 200 & 2.0 & 0.2 & 1 & 0.056 & 0.048 & 0.044 \\
2000 & 4.00 & 0.25 & 400 & 3.0 & 0.1 & 1 & 0.053 & 0.049 & 0.039 \\
2000 & 4.00 & 0.25 & 400 & 3.0 & 0.2 & 1 & 0.062 & 0.057 & 0.053 \\
2000 & 4.00 & 0.5 & 400 & 3.0 & 0.1 & 1 & 0.061 & 0.055 & 0.039 \\
2000 & 5.15 & 0.5 & 200 & 1.5 & 0.2 & 1 & 0.052 & 0.044 & 0.040 \\
2000 & 4.00 & 0.25 & 200 & 3.0 & 0.1 & 1 & 0.090 & 0.081 & 0.047 \\
2000 & 6.50 & 0.25 & 200 & 1.5 & 0.1 & 1 & 0.011 & 0.010 & 0.006 \\
2000 & 7.20 & 1 & 100 & 1.5 & 0.1 & 1 & 0.013 & 0.010 & 0.007 \\
\hline
\end{tabular}

\section{Creation of the Spike-in Data for Section 6.1}

We first generate a comprehensive spike-in dataset as follows: A $10 \mathrm{Mbp}$ region is randomly selected from the UCSC curated human reference genome version hg19 (Karolchik et al., 2013). Mutated versions of this region were generated, with each version containing 100 instances of insertions and deletions, of sizes between 50 and 10000 base pairs placed at random positions within the region. The dwgsim program in the DNA Analysis package (an improved version of wgsim of the SAMtools package (Li et al., 2009)) is then used to simulate Illumina reads with random point mutations and sequencing errors from each of the normal and mutant versions of the sequence. The normal reads and the mutant reads are combined at 100\%, 50\% and $25 \%$ mixing fractions, for each type and size of SV. In dwgsim, we set the read length to $100 \mathrm{bp}$, coverage to $50 \mathrm{x}$, mean insert size to $300 \mathrm{bp}$ and insert size standard deviation to 30bp. More details on the spike-in procedure are pro- 

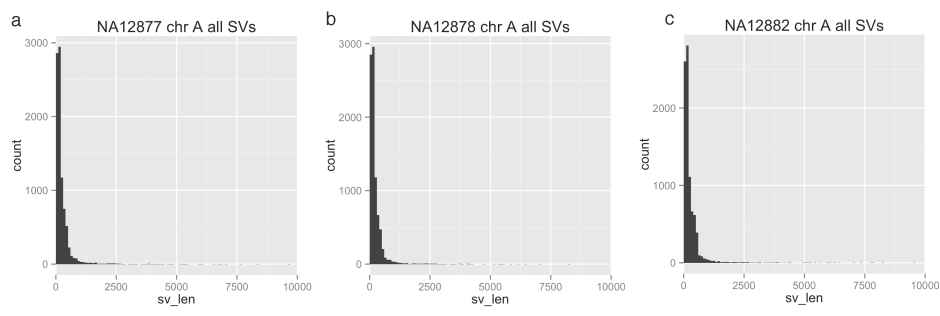

Figure 1: Sizes of Variants Detected in Illumina Platinum Genomes Data

vided in Fig. S2. Finally, we used the BWA aligner (Li et al., 2009) to map simulated reads back to the reference sequence, resulting in BAM files that are inputs to the SV callers.

\section{Summaries of Real Sequencing Data Sets}

Table 2: Features of Several Public Data Sets

\begin{tabular}{l|l|l|c|c|c|c}
\hline Source & Sample name & Date & $R$ & $\hat{\delta}$ & $\hat{\sigma}$ & $\hat{p}$ \\
\hline 1000 Genomes & NA12878 & Nov, 2011 & 36 & 197 & 9.6 & 0.01 \\
1000 Genomes & NA12878 & July, 2013 & 100 & 398 & 33 & 0.01 \\
1000 Genomes & NA12891 & July, 2013 & 100 & 342 & 70 & 0.01 \\
Illumina Platinum Genomes & NA12878 & July, 2013 & 100 & 313 & 76 & 0.03 \\
Illumina Platinum Genomes & NA12877 & July, 2013 & 100 & 319 & 76 & 0.03 \\
Illumina Platinum Genomes & NA12882 & July, 2013 & 100 & 314 & 76 & 0.03 \\
\hline
\end{tabular}

